Jean-François Hardy MD FRCPC,
Michèle Pelletier MD MSc†

\title{
La qualité en anesthésie: un modèle intégré et constructif
}

La gestion de la qualité a longtemps été perçue par les médecins comme une obligation administrative visant à corriger les comportements déviants. De nos jours, en ces temps de réorganisation de la pratique médicale et hospitalière, la gestion de la qualité est devenue essentielle au progrès de l'anesthésie-réanimation. ${ }^{1}$ D'ailleurs, les anesthésistes canadiens ont une longueur d'avance sur leurs collègues d'autres pays quant à la conceptualisation et la mise en application de modèles de qualité en anesthésie. ${ }^{2}$

Après un bref survol historique, cette conférence d'actualisation propose une définition de la qualité en médecine et décrit les approches existantes. Enfin, le modèle retenu insiste sur l'intégration de ces approches trop souvent indépendantes jusqu'à maintenant. Le modèle proposé est constructif et encourage chaque anesthésiste à faire de la gestion de la qualité une de ses priorités quotidiennes.

\section{Historique de la gestion de la qualité}

Pour mieux comprendre comment les concepts actuels se sont développés, à partir des succès et des écueils des différentes méthodologies appliquées à l'industrie, à la médecine ou à l'anesthésie, il est important de revoir brièvement l'historique de la gestion de la qualité.

Le célèbre code d'Hammourabi (1793-1750 av. J.C.), retrouvé à Suse en 1902 et conservé au Louvre, nous a permis de réaliser que, dès cette époque, la pratique de la médecine était réglementée et que des pénalités étaient prévues en cas de négligence. Les principes d'éthique avancés par Hippocrate (460-377 av. J.C.) nous apprennent, entre autres, à questionner l'efficacité de certains traitements à la mode. Plus près de nous, en 1342, le maire de Londres nommait deux Maîtres Chirurgiens avec mission de surveiller la pratique de leur art et de lui rapporter les collègues dont la pratique serait déficiente. Enfin, en 1518, le Collège Royal publiait ses normes de pratique, pour l'honneur des médecins et le bénéfice du public.

De cette gestion de la qualité dans une société artisanale, nous sommes passés à la gestion de la qualité dans une société industrielle. Frederick Winslow Taylor, ingénieur et économiste américain de la fin du XIX ${ }^{\circ}$ siècle, décrivait la qualité par l'inspection. Selon cette théorie, c'est l'inspecteur qui est directement responsable de la qualité d'un bien ou d'un service. Par la suite, au début des années 30 , W.A. Shewart précisait les méthodes d'analyse statistique du contrôle de la production visant à produire des produits standardisés de haute qualité. Ce n'est qu'en 1966 que A. Donabedian décrivait le premier modèle d'évaluation de la qualité appliqué aux soins de santé, basé sur la triade bien connue comprenant «Structure, Processus et Issue ». Durant cette même période, à l'autre bout du Pacifique, une révolution s'opérait. Réalisant que l'inspection produisait des résultats insatisfaisants, deux théoriciens américains, W. Edwards Deming et Joseph M. Juran mettaient au point les nouvelles méthodes d'amélioration continue de la qualité qui ont permis à l'industrie japonaise de faire trembler les Etats-Unis. Ce concept, basé sur l'amélioration des processus, n'a été appliqué en médecine que vers la fin des années 80 . Mais, comme l'amélioration continue de la qualité ne produisait pas toujours à elle seule les résultats escomptés, le concept de « benchmarking " a été introduit au début des années 90. L'étalonnage vise à évaluer la performance en fonction de références internes et/ou externes, redonnant son importance à l'amélioration des résultats plutôt que des seuls processus.

Au Québec, le concept d'amélioration continue se retrouve au centre de la gestion de la qualité de l'exercice professionnel mise de l'avant par le Collège des médecins depuis $1994 .^{3}$ Par ailleurs, plusieurs outils statistiques permettent de comparer la performance des institutions au sein de groupes d'établissements de vocation et de taille semblables.

Mais, au fait, qu'est-ce que la qualité?

Intuitivement, nous savons tous ce qu'est la qualité. La définir est beaucoup plus difficile probablement parce que, comme la beauté, la qualité dépend de la perspective de l'évaluateur. Le dictionnaire Larousse définit la

\footnotetext{
* Département d'anesthésie-réanimation, Institut de Cardiologie de Montréal, Université de Montréal, Qc.

†Directeur des services professionnels, C.H. St-Eustache, Qc.
} 
qualité ainsi: 1) une manière d'être, un état caractéristique; 2) supériorité, excellence en quelque chose et 3 ) ce qui fait le mérite de quelqu'un. ${ }^{4}$ Chose certaine, dans le domaine médical, la notion de qualité a une connotation nettement positive, désirable et recherchée.

Certaines définitions formelles ont été proposées, $2,3,5,6$ mais il est plus pratique de définir la qualité en fonction des attributs de l'acte médical. La définition des attributs recherchés est à la fois beaucoup plus simple, facile à comprendre, et essentielle à la mise en place d'activités visant à évaluer et améliorer la qualité. ${ }^{2,6} \mathrm{Bien}$ sûr, ces attributs varient en fonction de la clientèle interrogée mais, en anesthésie, tous les évaluateurs s'entendent sur au moins deux points. D'abord, les normes retenues devront réfléter la qualité de vie élevée de notre société. Ensuite, il est évident que l'incidence de mortalité ou de morbidité anesthésique majeure doit être minime.

Mais pourquoi se préoccuper de qualité en anesthésie? ${ }^{1}$ Une pratique de qualité ne nous apporte aucun avantage financier ni, contrairement à plusieurs autres spécialités, aucune augmentation du nombre de patients référés. Par contre, une pratique de qualité augmente la valeur du département aux yeux de nos clients (ici le terme client est pris au sens large du terme et englobe les personnes qui interagissent avec' le département d'anesthésie). Ces clients, ce sont nos patients, nos collègues chirurgiens, nos administrateurs et nos étudiants le cas échéant. Comme dans tout autre domaine, l'appui de nos clientèles passe par leur satisfaction. Cet appui de nos clientèles est essentiel au progrès de l'anesthésieréanimation, surtout en ces temps de restrictions budgétaires et de réorganisation de la pratique médicale et hospitalière.

Aux yeux du patient, l'anesthésiste est (le plus souvent) un inconnu et l'anesthésie est un mal nécessaire plutôt qu'un traitement. Comme pour tous nos autres clients, l'absence de mortalité ou de morbidité sévère est primordiale. Cependant, l'incidence de ces évènements défavorables est maintenant si rare que les effets secondaires sont devenus une source de préoccupation plus palpable pour nos malades. L'amélioration de la qualité passe donc par le contrôle des effets secondaires les plus fréquents (nausées/vomissements, céphalées, maux de gorge), même s'ils peuvent nous apparaître mineurs. ${ }^{7}$

Aux yeux du chirurgien, l'anesthésiste est un facilitateur. Dans cette perspective, le rôle de l'anesthésiste sera de s'assurer du bon déroulement des activités au bloc opératoire, que le patient arrive en salle d'opération dans les meilleures conditions possibles, que la stabilité intraopératoire est optimale et l'évolution postopératoire sans accroc.

L'administrateur et le système de santé définissent la qualité en médecine par un certain nombre d'attributs. ${ }^{3}$ Ceux-ci s'appliquent également très bien à l'anesthésie. ${ }^{2}$ Brièvement, ces attributs sont:

- la sécurité: le risque est minimal pour le patient

- l'efficacité: les services produisent les résultats escomptés

- la compétence: des individus qui dispensent le service

- l'acceptabilité: vs les attentes des patients, des collègues et de l'accréditation

- la disponibilité: des individus qui dispensent le service

- les services sont appropriés: aux besoins et aux idéaux de l'institution

- l'efficience: les services sont rendus au meilleur coût.

Enfin, aux yeux de l'étudiant, l'anesthésiste est, du moins au départ, un inconnu et l'anesthésie n'est pas un traitement. Il faudra donc l'intéresser par un enseignement théorique et une formation pratique bien adaptés à son niveau de connaissances. L'intégration à l'équipe soignante ainsi que le rôle modèle de l'anesthésiste et de son département favoriseront l'apprentissage de l'étudiant tout en assurant à notre spécialité considération et respect.

La gestion de la qualité en médecine: inspection ou amélioration continue?

Les médecins revoient et discutent, de longue date, les évènements catastrophiques afin d'en tirer les enseignements qui s'imposent. Dans la plupart des milieux, cette revue prend la forme d'une réunion dite de " morbiditémortalité ", dure environ une heure par mois et, dans les cas extrêmes, permet quelques échanges musclés entre les protagonistes.

L'assurance de la qualité, une activité plus structurée permettant la revue périodique des activités, est bien connue de nos voisins américains. ${ }^{8}$ L'assurance de la qualité étudie.la structure dans laquelle nous travaillons, (e.g., la conformité des machines d'anesthésie) les processus régissant nos activités (e.g., l'identification d'un malade à son arrivée au bloc opératoire), et l'issue des soins prodigués (e.g., l'absence de complications anesthésiques). Divers indicateurs permettent au chef de département d'évaluer la performance des membres de son département et d'émettre les recommandations pertinentes quant au renouvellement de leurs privilèges, avec ou sans conditions. ${ }^{9}$ Les limitations de l'assurance de la qualité ainsi définie sont immédiatement apparentes. La revue des activités s'attarde principalement aux évènements négatifs, met l'emphase sur le rôle déterminant des personnes, et est donc perçue comme coercitive et punitive. Cette façon de faire est démoralisante et ne stimule ni l'enthousiasme ni la participation des membres du département. De plus, ces activités 
intra-départementales, à visée normative, ne favorisent pas les interactions avec le reste de l'organisation.

Au Québec ces limitations ont handicapé l'essor de ce type de gestion de la qualité au niveau départemental. Ainsi, le « Guide de l'évaluation des actes médicaux par critères objectifs » publié en 1983 par la Corporation professionnelle des médecins du Québec a eu peu de suites en pratique clinique. Néanmoins, au niveau institutionnel, tant au Canada et qu'au Québec, les organismes accréditeurs pratiquent couramment des activités d'assurance de la qualité, par leurs exigences réglementaires touchant les structures et les processus en place dans les milieux évalués.

La philosophie et le système de gestion de l'activité médicale mis de l'avant depuis le début des années 90 par l'amélioration de la qualité sont entièrement différents. Tout le personnel hospitalier est impliqué, du conseil d'administration au plus bas salarié. L'amélioration recherchée est continue et globale, favorisant le déplacement vers le haut de toute la courbe de performance et non de ses seules extrémités. Enfin, et la différence est de taille, l'amélioration de la qualité met l'emphase sur le processus plutôt que sur les individus en affirmant que ce sont les processus qui sont imparfaits et non les individus oeuvrant au sein de l'organisation qui manquent de motivation, de connaissances ou d'habiletés. L'individu se sentant ainsi valorisé et écouté participe plus volontiers à l'amélioration de la qualité car, comme la plupart d'entre nous, il aspire à un travail bien fait.

Ces antagonismes entre l'assurance de la qualité (l'individu est au coeur du problème: la « Theory of Bad Apples ") et l'amélioration continue de la qualité (le système peut être amélioré: la « Theory of Continuous Improvement ») ont été remarquablement décrits et illustrés par Donald M. Berwick. ${ }^{10}$

\section{Un modèle intégré et constructif}

Afin de pallier aux limitations individuelles des différentes approches décrites précédemment, la Figure 1 présente un modèle qui intègre à la fois la collecte des données, la gestion du risque, l'assurance de la qualité, l'amélioration continue de la qualité et, finalement, la gestion des ressources. La description de chacun de ces éléments souligne sa complémentarité avec les autres composantes du modèle.

\section{La collecte et la présentation des données}

La gestion de la qualité ne peut se faire sans information sur les attributs que nous désirons évaluer. Une information objective, précise, claire et bien présentée est donc le pivot central autour duquel s'articuleront toutes les autres composantes du système. Egalement, afin

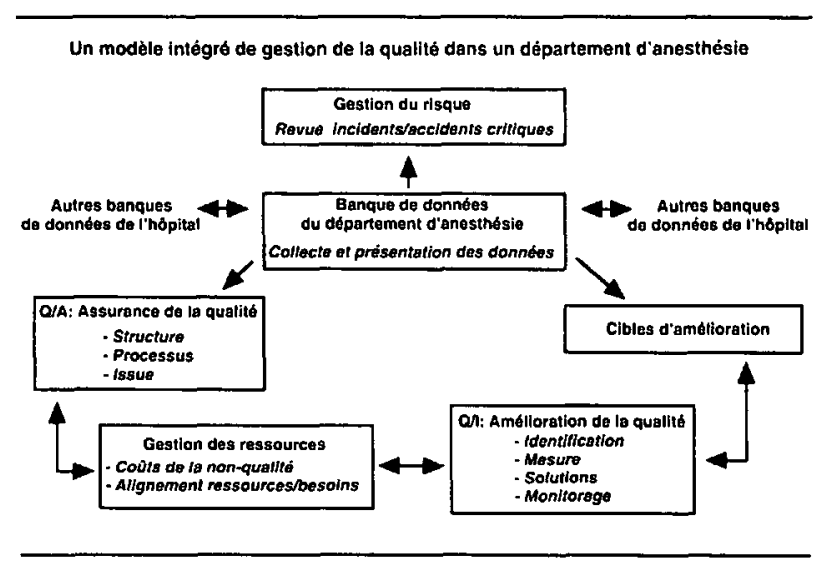

FIGURE 1 Intégration fonctionnelle des différentes composantes de la gestion de la qualité; inspiré de la référence 2.

d'éviter les dédoublements, tant au niveau de la collecte des données qu'au niveau des activités qui en découlent, il est important que l'information soit disponible à tous les départements concernés. Ainsi, par exemple, les banques de données de différents départements devraient être reliées entre elles. L'informatisation croissante de nos institutions favorise l'accès à l'information requise.

Classiquement, chaque anesthésiste rapporte ses cas difficiles qui pourraient faire l'objet d'une discussion aux réunions mensuelles de " morbidité-mortalité ». Cette source d'information est évidemment biaisée et incomplète, lorsqu'elle existe. Un échantillonnage plus formel, effectué par le service des archives de l'hôpital sur la base de critères prédéterminés (e.g., tous les malades opérés nécessitant un anti-émétique à la salle de réveil) fournit des renseignements précieux au département et permet d'étudier de grandes séries de manière efficiente. La revue de dossiers individuels demeure essentielle dans les cas plus rares. Enfin, la Loi sur la Santé et les Services Sociaux prévoit que certains dossiers doivent être revus obligatoirement, e.g. lorsqu'un patient décède à l'hôpital.

De plus, dans la " perspective clientèle » énoncée précédemment, nous devrons créer de nouveaux outils pour mesurer la perception de nos patients, de nos collègues et de nos étudiants. Ces outils pourraient prendre la forme d'entrevues avec les clientèles visées et/ou de questionnaires anonymes. Les informations recueillies apportent un éclairage différent sur notre pratique et nous permettent d'identifier des cibles d'amélioration non perceptibles par la seule revue des actes médicaux. A nouveau, tous ces renseignements devront être colligés dans une banque de données pour fins d'analyse.

La gestion du risque

La gestion du risque est cette composante de la gestion 
La gestion du risque: un modèle d'investigation d'accident anesthésique

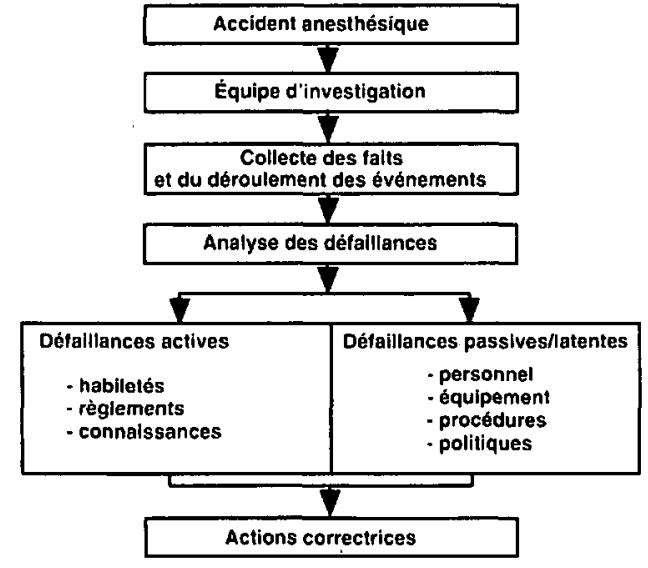

FIGURE 2 Un modèle de gestion du risque qui souligne l'importance des défaillances passives/latentes. Traduit de la référence 12.

de la qualité qui permet de revoir rapidement les incidents (l'évènement aurait pu être grave) ou les accidents (il s'est effectivement produit quelque chose de sérieux) critiques afin d'apporter les correctifs nécessaires dans les meilleurs délais. Encore là, diverses approches ont été proposées pour gérer les risques associés à l'anesthésie. Ces approches ressemblent plus ou moins à celles utilisées dans le domaine de l'aviation militaire. ${ }^{11}$

En 1992, Eagle $e t$ al. adaptait à l'anesthésie les nouvelles méthodes d'investigation de désastres et de leur causalité développées dans les complexes industriels à la suite d'une série d'accidents majeurs. ${ }^{12} \mathrm{Ce}$ modèle d'investigation des accidents anesthésiques est présenté à la Figure 2. Le plus tôt possible après l'accident, une équipe d'investigation est mise sur pied. La collecte détaillée des faits et du déroulement des évènements permettra d'analyser les défaillances qui ont mené à l'accident. L'originalité du modèle est qu'il distingue entre les défaillances actives (e.g., les gestes posés par l'anesthésiste) et les défaillances passives qui ont mené l'anesthésiste à poser ces gestes inadéquats dans les circonstances. ${ }^{13}$

Habituellement, l'emphase est mise sur les défaillances actives. Celles-ci sont faciles à identifier et le coupable peut être châtié rapidement. L'anesthésiste peut ne pas avoir eu les habiletés ou les connaissances requises, et/ou avoir enfreint une règle de pratique. Par contre, il faut également tenir compte des défaillances passives mais celles-ci sont difficiles à identifier et à corriger car c'est le système lui-même qu'il faut analyser et corriger. Ainsi, la formation du personnel de soutien, l'entretien de l'équipement, les politiques et les procédures en vigueur peuvent avoir contribué à l'accident. Dans l'exemple présenté par Eagle et al., la non- disponibilité de renseignements au dossier, l'absence de visite préopératoire, la procédure d'assignation des cas en salle d'opération sont autant de défaillances passives qui ont contribué à la pneumopathie d'inhalation. Bien sûr, l'anesthésie était une condition nécessaire (mais non suffisante) pour que l'accident survienne. La correction des défaillances passives évitera de placer à nouveau l'anesthésiste compétent dans le contexte favorisant l'erreur et, par le fait même, la répétition de ce type accident.

\section{L'assurance de la qualité}

L'assurance de la qualité revoit la structure, le processus et l'issue des activités courantes en fonction de normes reconnues et vise à corriger les lacunes détectées, mais ne cherche pas spécifiquement à améliorer les situations existantes. Dans la forme que nous lui connaissons en général au Canada, elle est surtout un outil de surveillance de la qualité des structures et des processus par les organismes accréditeurs. Cette évaluation externe d'un département ou d'une institution est une composante essentielle de la gestion de la qualité. Les activités d'assurance de la qualité nécessitent la collecte de quantités importantes d'informations qui seront comparées avec des normes internes et externes. Ainsi, c'est la composante du système qui génère les changements les plus lents.

L'assurance de la qualité peut être requise par les organismes accréditeurs (e.g., le Conseil canadien d'agrément des hôpitaux) ou par les pairs. Il existe au moins deux exemples de programmes d'assurance de la qualité initiés par des pairs. Aux Etats-Unis, l'American Society of Anesthesiologists a mis sur pied un programme de consultation (Anesthesia Consultation Program) permettant aux petits départements qui n'ont pas les ressources nécessaires sur place de se faire évaluer par des pairs. ${ }^{14}$ En Nouvelle-Ecosse, un programme d'évaluation des départements d'anesthésie par des pairs a été mis sur pied en 1987 afin de répondre aux attentes des anesthésistes et des médecins de la province. ${ }^{15}$ Entre 1987 et juin 1993, 22 départements ont été évalués au moins une fois et 4 départements l'ont été deux fois. Les carences les plus fréquentes concernaient, par exemple, la tenue des dossiers, la conformité des appareils $\mathrm{d}$ 'anesthésie aux normes en vigueur et la documentation d'activités d'assurance de la qualité. Fait intéressant, lors d'une seconde visite les carences initiales avaient été corrigées mais de nouvelles, absentes initialement, étaient apparues. Ce phénomène s'expliquerait par le couplage serré des activités dans le milieu hospitalier: toute modification, même mineure, dans une sphère d'activité retentit sur les autres. Ceci suggère qu'il est essentiel d'assurer le suivi après un changement. ${ }^{16}$ 
L'issue se mesure le plus souvent en fonction de critères tels que le décès, la maladie, l'incapacité, l'inconfort, l'insatisfaction ou les coûts secondaires à une intervention thérapeutique. En anesthésie, l'issue est plus difficile à évaluer car, règle générale, il ne s'agit pas d'un traitement. L'évaluation du processus (le " comment ") prend donc une place plus importante. En fait, nombreux sont ceux qui estiment que si les structures et les processus sont adéquats, l'issue sera forcément favorable. Non seulement est-ce présumer de l'issue de l'acte médical, mais c'est également négliger la perception de nos clientèles. Des nausées, des vomissements, des céphalées, des maux de gorge, une hospitalisation imprévue après une chirurgie mineure sont autant d'issues défarorables aux yeux de nos patients, même si l'anesthésie était impeccable techniquement.

\section{L'amélioration de la qualité}

L'amélioration continue de la qualité cherche spécifiquement à améliorer les situations existantes, contrairement à l'assurance de la qualité qui évalue les activités courantes en fonction de normes reconnues. Les activités d'amélioration de la qualité sont basées sur le cycle «planification-mise en place-vérificationrétroaction »et produisent des résultats plus lentement que la gestion du risque, mais plus rapidement que l'assurance de la qualité car les problèmes étudiés sont plus spécifiques. Le Collège des médecins du Québec insiste sur la nécessité de définir les objectifs avec le plus de précision possible car, de cette précision dépend souvent un programme d'activités d'amélioration de la qualité qui fonctionne et qui correspond aux problèmes réels de l'établissement. ${ }^{3}$

L'amélioration de la qualité repose sur 4 principes de base. Rappelons les:

- le système, non les personnes, est responsable de la qualité

- la qualité est l'affaire de tous

- la qualité est fonction de la perception de la clientèle visée

- la non-qualité coûte cher.

Trois types d'outils sont utilisés, principalement pour colliger et organiser les données, pour présenter les données et, enfin, pour tenter de comprendre le processus à l'étude.

En milieu hospitalier, l'amélioration de la qualité implique tout d'abord le maintien du système en place. ${ }^{6}$ Ces activités d'entretien permettront de distinguer entre les problèmes induits par des causes externes et les problèmes générés par le système lui-même. Les phénomènes extrinsèques produisent des variations importantes et inhabituelles des taux d'évènements défavorables. Le graphique de la Figure 3 pourrait

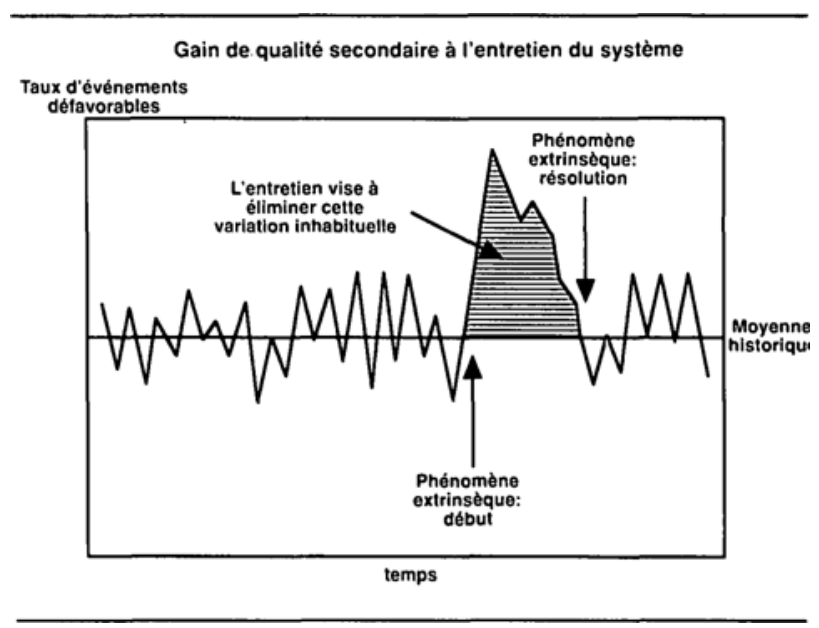

FIGURE 3 L'entretien du système permet d'identifier et de corriger les anomalies induites par un phénomène extrinsèque mais ne permet pas d'améliorer le système: le taux d'évènements défavorables demeure constant. Traduit de la référence 6.

représenter, par exemple, l'administration criminelle d'un médicament (e.g., bloqueur neuro-musculaire, morphinique) au bloc opératoire. La résolution de l'activité criminelle se traduira par un retour aux fluctuations de base du système en équilibre. Ces activités s'apparentent à celles de l'assurance de la qualité car elles garantissent la stabilité du système. Il a été suggéré que le système de soins lui-même est le déterminant majeur de la qualité des services anesthésiques, plus que l'anesthésiste traitant. ${ }^{17}$ Dans cette perspective, le maintien et l'amélioration du système revêtent une importance toute particulière.

Les activités d'amélioration (à proprement parler) du système verront à diagnostiquer les problèmes et apporter les correctifs appropriés. ${ }^{6}$ Ensuite, il faudra évaluer si les changements désirés ont bel et bien été apportés au système, et s'ils sont efficaces. La Figure 4 illustre les gains considérables obtenus à long terme par l'amélioration du système, comparativement aux gains obtenus par la simple correction d'une anomalie ponctuelle (Figure 3). La taille des systèmes et la lenteur des processus peut rendre certaines interprétations difficiles. Ainsi, il faudra s'assurer que les modifications de l'issue ne correspondent pas simplement à des modifications des entrées (input) dans le système (e.g., population vieillissante et plus malade ou, au contraire, plus jeune et en santé).

La Figure 5 illustre bien l'utilité des statistiques et la nécessité de les interpréter correctement et avec prudence. Le graphique illustre le nombre de traitements inappropriés (axe vertical) en fonction du nombre de traitements administrés (axe horizontal) pour un groupe de médecins qui ont traité une pathologie donnée. Tel 
Gain de qualité secondaire à l'amélioration du système

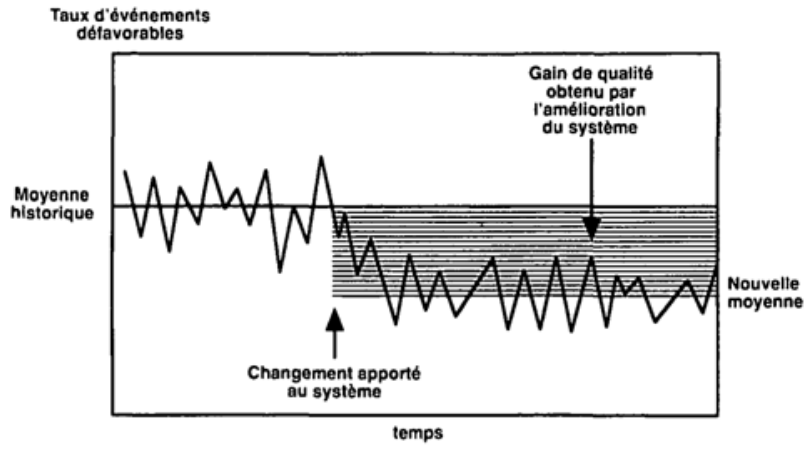

FIGURE 4 Les activités d'amélioration de la qualité permettent d'effectuer des gains à long terme en diminuant le taux moyen d'évènements défavorables de manière soutenue. Traduit de la reférence 6.
Analyse statistique de la performance médicale

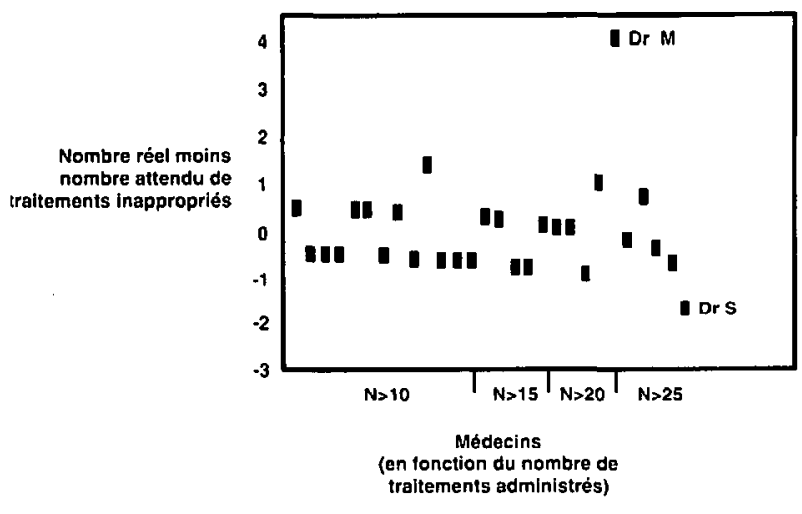

FIGURE 5 L'analyse statistique de la performance médicale permet d'identifier certaines tendances. Les statistiques du Dr M. indiquent une performance Médiocre qui mérite d'être investiguée et corrigée si l'investigation confirme que ses traitements sont inappropriés. Mais le système gagnera beaucoup plus en ramenant la performance de tous à celle du Dr S. dont les résultats sont nettement Supérieurs à ceux de ses collègues. Traduit et adapté de la référence 6.

que prévu, la moyenne des individus se situe autour de zéro, c'est à dire que leur niveau de performance était celui anticipé. Deux individus ressortent nettement, les Drs M. et S. Le Dr M. pourrait être qualifié de médiocre à l'examen du graphique, mais il existait peut-être des circonstances cliniques spéciales justifiant cette observation. Il est important de réaliser que l'étude statistique nous indique qu'un problème peut exister, mais qu'elle ne peut poser de diagnostic précis. Autrement dit, il s'agit d'un indicateur et non d'un déterminant de la qualité. Contrairement au Dr M., la performance du Dr $\mathrm{S}$. apparait supérieure à celle de ses collègues. Si cela se confirme après étude, le système gagnera donc beaucoup plus à amener la moyenne globale à celle du Dr S. qu'à ramener le Dr M. au sein du peloton (ce qui doit aussi être fait sans faute, dans le cas où ses traitements étaient véritablement inadéquats).

\section{La gestion des ressources}

La non-qualité coûte cher en temps perdu pour les patients, pour les médecins et pour l'hôpital, en perte de revenus et de productivité pour les employeurs, en traitement des complications à domicile ou en institution et, à l'occasion, en dépenses d'ordre médico-légal. Règle générale, tous ont donc un intérêt immédiat à offrir les services de la plus haute qualité possible.

Un des objectifs importants de la gestion de la qualité est l'alignement des ressources disponibles sur les besoins en santé. En ces temps de compressions budgétaires, les ressources sont de plus en plus limitées, alors que la technologie nous propose de nouvelles molécules ou de nouveaux appareils de diagnostic et de traitement, plus performants mais aussi souvent plus chers. Selon Dexter et Tinker, la qualité de l'anesthésie est déjà telle qu'il n'y aurait pas d'avantage financier à l'améliorer en ce qui concerne les quatre chirurgies à risque modéré analysées dans leur étude (transplantation rénale, césarienne, prothèse totale de hanche, cholécystectomie par coelioscopie). ${ }^{18}$ Ces auteurs ont sans doute raison, mais seulement en partie. Il est indiscutable que, grâce aux nouveaux médicaments et au monitorage de plus en plus poussé des fonctions vitales, l'anesthésie est devenue extrêmement sécuritaire. Mais, si l'on se replace dans la perspective clientèle décrite précédemment, de nombreuses améliorations restent à faire. Par exemple, une anesthésie optimale n'affectera que très brièvement le patient subissant une chirurgie mineure en court séjour et pourra entraîner des économies substantielles tant pour l'individu concerné que pour sa famille et la communauté. ${ }^{19}$

La gestion de la qualité elle-même coûte cher et, en anesthésie, peut paraître difficile à justifier sur des bases purement économiques. ${ }^{20}$ Seuls quatre items ont produit une économie réelle sur les 53 projets de gestion de la qualité rapportés par Eagle et al. et les revenus générés n'assuraient pas l'auto-financement du programme. ${ }^{20}$ Devrait-on pour autant abandonner ces activités? Non, évidemment, pour plusieurs raisons: ${ }^{21}$ Contrairement aux spécialités à visée thérapeutique, en anesthésie, il est difficile de mesurer les gains financiers de l'amélioration de la qualité avec des indicateurs reconnus tels que la longévité en bonne santé (QALY: Quality Adjusted Life Years). De nouveaux outils de mesure des 
avantages financiers découlant de la gestion de la qualité devront être mis au point spécifiquement pour nous. Par ailleurs, le fait de travailler dans un environnement financièrement peu compétitif rend les avantages monétaires d'une anesthésie de qualité moins évidents. ${ }^{1}$

Les conséquences de la non-gestion de la qualité par les médecins responsables seraient majeures pour notre spécialité. Vu l'intérêt croissant porté par le public aux services médicaux et compte tenu du besoin inchangé de restreindre les dépenses, un organisme de contrôle externe au département serait sans aucun doute mis sur pied pour évaluer notre pratique et mettre en place des protocoles d'évaluation et de traitement. Aux EtatsUnis, de nombreuses directives de pratique (practice guidelines) ont été élaborées pour tenter de restreindre les écarts de pratique entre médecins et, par voie deconséquence, contrôler les coûts du système de santé. ${ }^{22}$ Nous avons la chance de pratiquer dans un système où le médecin est encore autonome. Seule une pratique de qualité dans le respect des contingences économiques actuelles nous permettra de conserver cette autonomie.

\section{Conclusions}

La gestion de la qualité est une question complexe, qui doit être abordée sous plusieurs angles. Chacun des éléments décrits est essentiel au maintien, à l'amélioration continue et à la gestion des coûts de la qualité.

Ce texte encourage chaque anesthésiste à faire de la gestion de la qualité une de ses priorités quotidiennes. En quelque sorte, il s'agit là du « pourquoi » de la gestion de la qualité de l'exercice professionnel. L'application des principes mis de l'avant (le « comment ») prendra une forme différente selon le milieu et/ou le pays où l'anesthésiste pratique. Au Québec, en établissement, le lecteur est référé au document publié par le Collège des médecins. ${ }^{3} \mathrm{Ce}$ document de référence décrit bien les fondements légaux, les mécanismes et les outils de travail pertinents dans notre contexte.

La qualité de la recherche anesthésique est un sujet distinct mais dont l'impact est primordial car la recherche fondamentale et clinique représentent les assises de notre pratique clinique. Certains mécanismes, tels que l'attribution de fonds par les organismes subventionnaires et la revue de manuscrits par des comités de pairs, constituent déjà une forme d'assurance de la qualité. ${ }^{23}$ Cependant, il y aurait sans doute lieu de gérer la qualité en recherche de manière plus formelle. ${ }^{23} \mathrm{Le}$ modèle clinique décrit pourrait être facilement transposé en recherche.

A l'aube de ce troisième millénaire, seule une approche constructive, orientée vers le patient, en harmonie avec le milieu nous permettra d'atteindre notre objectif: assurer à chaque jour et à chacun de nos malades une anesthésie de qualité, à la fois sur le plan technique, humain et financier.

\section{Références}

1 Duncan P. Quality: a job well done! (Editorial) Can J Anaesth 1993; 40: 813-5.

2 Eagle CJ, Davies JM. Current models of "quality" - an introduction of anaesthetists. Can J Anaesth 1993; 40 : 851-62.

3 Corporation professionnelle des médecins du Québec. La gestion de la qualité de l'exercice professionnel en établissement. Montréal: 1994.

4 Le Petit Larousse illustré: dictionnaire encyclopédique. Paris: Larousse, 1992.

5 Laffel $G$, Blumenthal $D$. The case for using industrial quality management science in health care organizations. JAMA 1989; 262: 2869-73.

6 Kritchevsky $S B$, Simmons $B P$. Continuous quality improvement. Concepts and applications for physician care. JAMA 1991; 266: 1817-23.

7 Cohen MM, Duncan PG, Pope WD, et al. The Canadian four-centre study of anaesthetic outcomes: II. Can outcomes be used to assess the quality of anaesthesia care? Can J Anaesth 1992; 39: 430-9.

8 American Society of Anesthesiologists. Peer Review in Anesthesiology. Park Ridge, Ill.: 1992.

9 Vacanti CJ, Vitez TS. How quality assurance and the peer review process can help your department, $I n$ : Barash $\mathrm{P}$ (Ed.). ASA Refresher Courses in Anesthesiology. Peer Reviewed. Park Ridge, Ill.: 1992: 60-75.

10 Berwick DM. Sounding board. Continuous improvement as an ideal in health care. N Engl J Med 1989; 320: 53-6.

11 Davies JM, Lee RB, Cooper JB, Armstrong JN, McIntyre $R W$. Critical incidents in anaesthesia: medico-legal and other aspects (Symposium Report). Can J Anaesth 1991; 38: 1028-36.

12 Eagle CJ, Davies JM, Reason J. Accident analysis of large-scale technological disasters applied to an anaesthetic complication. Can J Anaesth 1992; 39: 118-22.

13 Duncan PG. Who's to blame? (Editorial). Can J Anaesth 1992; 39: 110-3.

14 Brown EM. Problem solving as a method of quality improvement in anesthesiology, In: ASA Refresher Courses in Anesthesiology. Peer Reviewed . Park Ridge, Ill.: 1992: 76-81.

15 Clark AJ. The Anaesthetic Services Programme Encompassing Nova Scotia (ASPENS). Can J Anaesth 1994; 41 : 716-24.

16 Davies JM, Priddy RE. Structural aspects of anaesthetic care (Editorial). Can J Anaesth 1994; 41: 661-6.

17 Lagasse RS, Steinberg ES, Katz RI, Saubermann AJ. Defining quality of perioperative care by statistical pro- 
cess control of adverse outcomes. Anesthesiology 1995;82:1181-8.

18 Dexter F, Tinker JH. The cost efficacy of hypothetically eliminating adverse anesthetic outcomes from high-risk, but neither low- or moderate-risk, surgical operations. Anesth Analg 1995; 81: 939-44.

19 Kapur PA. Cost containment: at what expense? (Editorial). Anesth Analg 1995; 81: 897-9.

20 Eagle CJ, Davies JM, Pagenkopf D. The cost of an established quality assurance programme: is it worth it? Can J Anaesth 1994; 41: 813-7.

21 Duncan PG. The price of quality. Can J Anaesth 1994; 41: 765-8.

22 Kassirer $J P$. The quality of care and the quality of measuring it (Editorial). N Engl J Med 1993; 329: 1263-5.

23 Sykes $M K$. Quality assurance in research. Eur J Anaesthesiol 1995; 12: 441-4. 
Jean-Françoị Hardy MD FRCPC, Michèle Pelletier MD MSc

\section{Quality in anaesthesia: an integrated and constructive model}

quality goods. More recently, A. Donabedian described a model of quality assessment, based on the well known triad of Structure, Process and Outcome. At the same time, a revolution was developing at the other end of the world. Two American thinkers W. Edward Deming and Joseph M. Juran, aware that inspection was not giving the expected results, completed the new methods of continuous quality improvement that allowed Japanese industry to shake the confidence of the US. This concept, based on the improvement of processes was finally applied to medicine in the late eighties. As continuous quality improvement by itself was not giving the expected results, the concept of "benchmarking" was introduced in the early nineties. Standardisation aims at assessment of performance relative to internal or external reference emphasising improvement of outcome rather than improvement of processes.

In Quebec, the concept of continuous improvement is now central to quality management of professional practice put forward by the College of Physicians since $1994 .^{3}$ Accordingly, many statistical tools allow to compare performance between establishments of identical size and vocation.

evolved from different methods as they apply industry, medicine and anaesthesia through their achievements and pitfalls.

The code of Hammurabi (1793-1750 BC) found at Susa in 1902 and kept at the Louvre Museum in Paris, has shown that medicine already had rules and punishment for negligence. The ethical principles proposed by Hippocrates (460-377 BC) teach us; amongst other things, to challenge the efficacy of certain fashionable cures. In 1342, the lord mayor of London designated two master surgeons to assess deviant practice. Finally, in 1518, the Royal College described standards of medicine being upheld for the probity of physicians and the benefit of the population.

Quality management evolved from these crude models of quality management to a refined model more appropriate to the industrial society. In the late 19th century, an engineer and economist, Frederick Winslow Taylor, reported on quality managed by inspection. According to his theory, the inspector is directly responsible for the quality of a product or service. In the early thirties, W.A. Shewart described a statistical analysis aiming at a standardised production control of high

\section{By the way, what is quality?}

We all know intuitively what quality is. To define it is a much more difficult task probably because quality is like beauty and thus depends on whoever is the judge. The Larousse dictionary defines quality as: (1) a way of being, a characteristic state; (2) superiority, excellence in a domain and (3) what confers the value to someone. ${ }^{4}$ In medicine, the notion of quality indisputably implies a positive, desirable and expected approach.

More formal definitions have been proposed ${ }^{2,3,5,6}$ but it is practical to define quality in terms of the attributes as they apply medical practice. The definition of desirable attributes vary according to the queried customers, but in anaesthesia, two elements are accepted unanimously by all. To be accepted the criteria should reflect, first, the high quality of living of our society and, second, a minimal incidence of mortality and morbidity.

Why bother with quality in anaesthesia? ${ }^{\text {Activities }}$ concerned with quality do not bring any monetary rewards and unlike other specialities, do not enhance the 
referral of new patients. On the other hand, quality practice enhances the value of a department to the eyes of its "customers" (the term "customer" has to be considered at its widest sense and includes everybody that interacts with the department of anaesthesia). The customers include patients, surgical colleagues, administrators and even students if any. The support of "customers" is essential to the improvement of anaesthesia and intensive care specially during periods of budget cuts and hospital reorganisation.

In the eyes of the patient, the anaesthetist is most of the time an anonymous being and anaesthesia is more a necessary evil than a means of treatment. As with all other "customers", the absence of mortality or severe morbidity is fundamental. However, the incidence of these adverse events has now become so rare that sideeffects have become the real cause of concern for patients. Thus quality improvement must now consider the control of the most frequent side-effects (nausea and vomiting, headaches, sore throat) although they may appear to be minor. ${ }^{7}$ For the surgeon, the anaesthetist is a facilitator. As such, the anaesthetist will see that the operating rooms are running smoothly, that the patient is at the best of conditions for his operation, that he is stable during surgery and that postoperative care is flawless.

The administrators and the health care system define quality in medicine with a number of attributes. ${ }^{3}$ These attributes apply to anaesthesia as well. They are:

- safety: the risk to patients is minimal

- effectiveness: the services are producing the expected results

- competence: the individuals performing the service

- acceptability: acceptation by the patients, colleagues and accredits

- accessibility: the performance of the service

- appropriateness: fulfilment of the needs and expectations of the institution

- efficiency: the services are delivered at the optimal cost.

In the eyes of the student, the anaesthetist may be a nobody and anaesthesia has no therapeutic virtue. His theoretical teaching and practical training must be adapted to his level of knowledge. His integration to the health care team and the enlightened performance of the anaesthetist and his department will be of great help in training students. Thus, it will also confer esteem and respect to our speciality.

Quality management in medicine: inspection or continuous improvement?

For many years, physicians in most centres have reviewed and discussed disasters because they wished to learn from them. This review process is a monthly morbidity-mortality forum lasting approximately one hour. In extreme cases, the discussion may be vigorous and passionate.

In the US, quality assurance is well recognised as a more structured method for reviewing clinical activities. ${ }^{8}$ It reflects on our professional environment (e.g., compliance of anaesthesia machines to standards), the processes regulating our activities (e.g., identification of patients arriving in the operating room), and the outcome of delivered care (e.g., absence of complications related to anaesthesia). With the help of various indicators, the head of department can assess the performance of his colleagues and make appropriate recommendations with regards to the granting of privileges. ${ }^{9}$ If we apply this definition, the limitations of quality assurance become obvious. This review of activities focuses mostly on negative aspects, emphasising the determinant role of individuals; it is therefore perceived as punitive and compelling. This method is hard on the morale and does nothing to stimulate enthusiasm and involvement from the staff. Furthermore, these intradepartemental activities aiming to comply with standards do not facilitate interactions with the rest of the institution.

In Quebec, these limitations have hindered the development of this type of quality management at the departmental level. Consequently, the guide for the evaluation of medical acts in relation with objective criteria published in 1983 by the Professional Corporation of Physicians had no effect on clinical practice. Nevertheless, at the institutional level, both in Quebec and in Canada, the accreditation councils are currently performing some of the activities relevant to quality assurance through established rules applying to structures and processes in the assessed centres.

The philosophy and management procedures adopted for medical practice in the early 90's by QI are totally different. The whole hospital staff is involved from the chairman of the board to the lowest ranked personnel. The expected improvement curve is continuous and global furthering the displacement of the whole performance curve toward the top and not only its extremities. Finally, and this difference is substantial, QI focuses on the processes rather than the individuals; indeed, it establishes that the processes themselves could be faulty rather than the individuals through their lack of drive, of knowledge or competence. The importance of the individual are thus increased and he is more willing to take part in the process of quality improvement, since he strives for a job well done like most of us do.

The conflict between quality assurance (the individual is central to the problem: the theory of "bad apples") and continuous quality improvement (the system can 


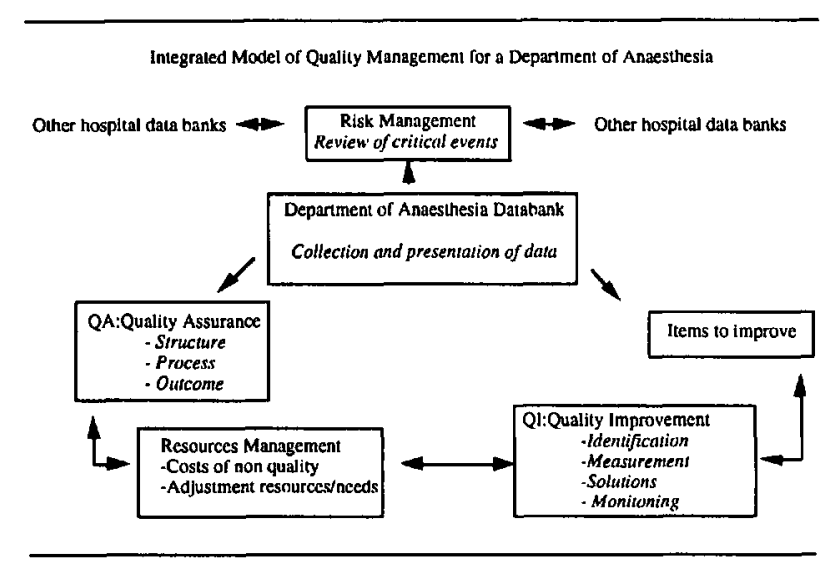

FIGURE 1 Functional integration of various components of quality management according to Reference 2 .

improved: "The theory of continuous improvement") are particularly well defined and pictured by Donald $\mathrm{M}$. Berwick. ${ }^{10}$

\section{An integrated and constructive model}

To offset the individual limits of the various approaches already described, Figure 1 outlines a model integrating data collection, risk management, quality assurance, continuous quality improvement and finally resources management.

\section{Collection and presentation of data}

Quality management cannot be performed without the knowledge of the attributes that we wish to assess. The information must be objective, precise, clear and well presented; it is the axis of the integration of other components to the system. To avoid duplication, both at the levels of data collection and of the ensuing activities, the information must be available to all the concerned departments. Therefore, for example, the banks of data of various departments should be interconnected. The increased computerisation of our institutions can facilitate the access to useful information.

In clinical practice, each anaesthetist reports the difficult cases that should be discussed at the monthly morbidity-mortality meeting. When it exists, this source of information is obviously biased and incomplete. A more rigorous sampling by the archive department of the hospital based on predetermined criteria (e.g., all the patients requiring anti-emetic drugs in the recovery room) provides the department with valuable information and allows large series to be studied efficiently. The review of individual files may be essential in certain cases. Finally, the health and social service legislation provides that certain files must be reviewed systematically (e.g., if a patient dies while in hospital).

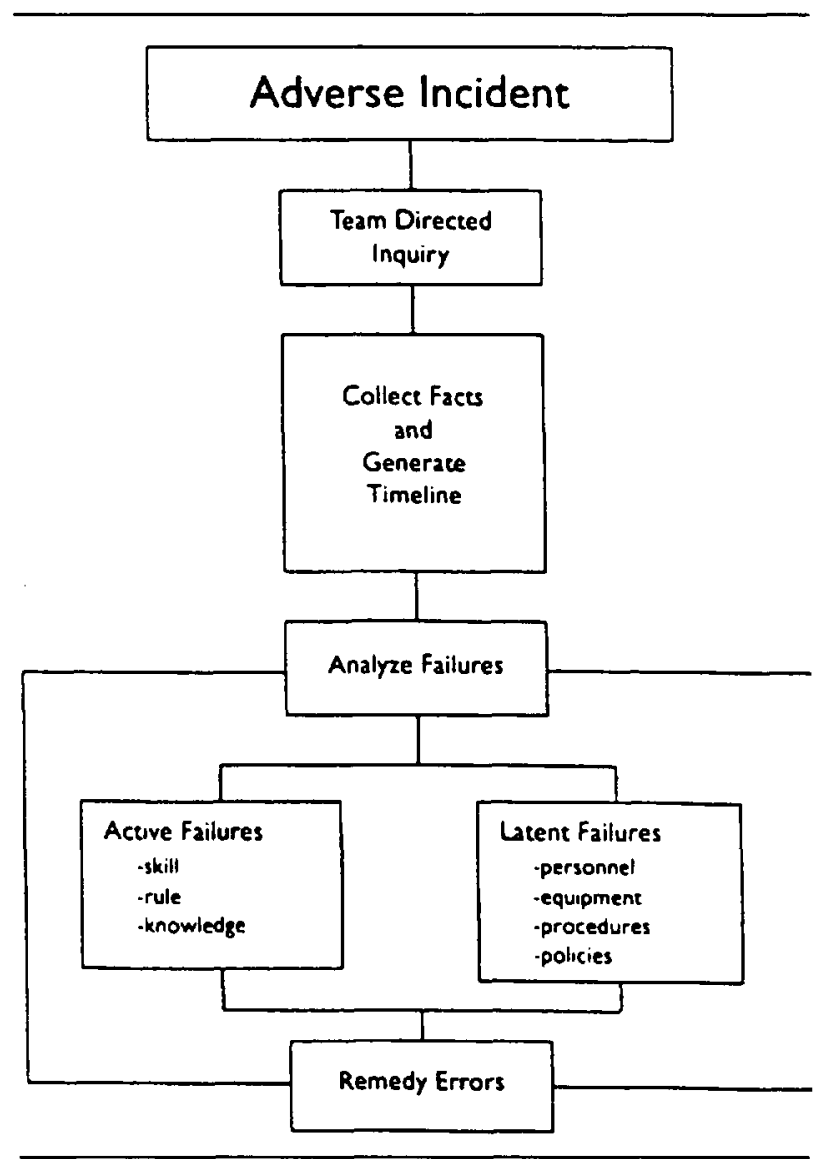

FIGURE 2 A model of accident investigation. Reference 12 in the text.

In addition, in the perspective of the customer previously expressed, we must create new tools to develop an opinion on the perception of patients, colleagues and students. These tools could consist of interviews with selected customers and/or of anonymous questionnaires. The collected information offers a different and clearer view of our practice and focuses on areas of improvement otherwise undetected. This information will also have to be integrated to a databank for further analysis.

\section{Risk management}

Risk management, a component of quality management allows a quick revision of mishaps (events that could have been serious) or accidents (events that were serious) for essential and urgent corrections. Various approaches to risk management in anaesthesia have been proposed. In some ways, they appear similar to the ones used in military aviation. ${ }^{\prime \prime}$

In 1992, the investigative methods developed for disasters occurring in industrial complexes and their relative causality were adapted to anaesthesia by Eagle et al. ${ }^{12}$ This new concept is presented on Figure 2. 
Following an accident, a team of investigators is promptly assembled. A careful collection of information concerning the accident and its circumstances will lead to the failures that caused it. This original model distinguishes the active failures (e.g., the anaesthetist's performance) from the latent failures which lead to an inadequate behaviour by the anaesthetist. ${ }^{13}$

Generally, active failures have received the focus of attention. They are easily to identified and the delinquent can be quickly punished. The anaesthetist may be inept or ignorant or/and may have broken a rule of practice. Conversely, latent failures must be considered but they are difficult to identify, as it is the system itself that must be examined and improved. Thus, the training of auxiliary personnel, the maintenance of equipment, the policies and proceedings could have been contributory to the accident. In the example reported by Eagle et al., the lack of information from the hospital file, the absence of a preoperative assessment, the operating room scheduling procedures were all latent failures leading to aspiration pneumonia. Anaesthesia was definitely an absolute condition for its occurrence, but the complication was still avoidable. In the future, the correction of latent failures will prevent placing the anaesthetist in an accident prone environment and therefore repeating the same type of mishap.

\section{Quality assurance (QA)}

Quality assurance relates structure, processes and outcome of current activities to established standards and aims at correcting the detected deficiencies but not necessarily at improving existing situations. In Canada, it is mostly a tool used by accrediting bodies to control the quality of structures and processes. This external review. of a department or institution is an essential component of QA. An extensive gathering of data is needed for the processes of QA to function, allowing the establishment of comparisons between external and internal standards. At the same time, the magnitude of the compilation is slowing down the whole process.

Quality assurance may be required by the accrediting bodies (e.g., the Canadian Council of Hospital Accreditation) or by peers. We know at least two examples of QA programmes instituted by peers. In the US, the American Society of anesthesiologists created the Anaesthesia Consultation Programme to allow the peer review of small departments with inadequate resources. ${ }^{14}$ In Nova Scotia, a peer review program was instituted in 1987 at the request of the anaesthetists and physicians of that province. Between 1987 and June 1993, 22 anaesthesia departments were inspected at least once and four departments were inspected twice. For instance, the most frequently encountered deficien- cies included non conformity with current standards of anaesthesia equipment, poor record keeping and absence of documentation of QA processes. Interestingly, at a second visit, some of the previously identified deficiencies had been corrected, but new ones had emerged. This could be explained by the tight integration of hospital processes; even the smallest change in one sector of activity has an effect on the other sectors. ${ }^{16}$

The outcome of every therapeutic intervention is measured in terms of mortality, morbidity, incapacity, discomfort, dissatisfaction and costs. As anaesthesia is not usually curative, its outcomes are very difficult to evaluate. The appraisal of processes ("how") is therefore very important. In fact, some believe that if structures and processes are adequate, the outcome has to be favourable. Not only this is a presumption on the outcome, but it also shows poor knowledge of customers' perception. Nausea, vomiting, headache, sore throat or unplanned hospital admission are significant adverse outcomes in the eyes of the patient, even if technically the anaesthesia was faultless.

\section{Quality improvement (QI)}

Continuous quality improvement aims specially at improving existing situations; by contrast, QA evaluates current activities in relation with accepted standards. The process of QI is based on the cycle of "planning doing - checking - acting (PDCA)." It produces results more slowly than risk management but more rapidly than QA as it relates to the investigation of more specific problems. The College of Physician of Quebec emphasises the necessity of a more precise definition of the objectives. A QI programme is often dependant on precision to function and to meet the real needs of the institution. $^{3}$

QI is based on the four following principles:

- it is the system that is responsible, not the persons working within it

- quality is everybody's business

- quality is relative to the "customer's" perception

- poor quality is costly

Three tools are mostly used to collect, organise and to present data, and to provide an insight on the process under study.

In the hospital environment, QI implies maintenance of existing system. ${ }^{6}$ The activities performed to maintain the system will allow to distinguish problems created by external causes from problems generated within the system. Extrinsic events cause unusual and considerable variations on the rate of adverse outcomes. For instance, the diagram on Figure 3 could be a representation of the criminal administration of a drug (e.g., muscular relaxant, narcotic) in the operating room. Resolv- 
Statistical Analysis of Medical Performance

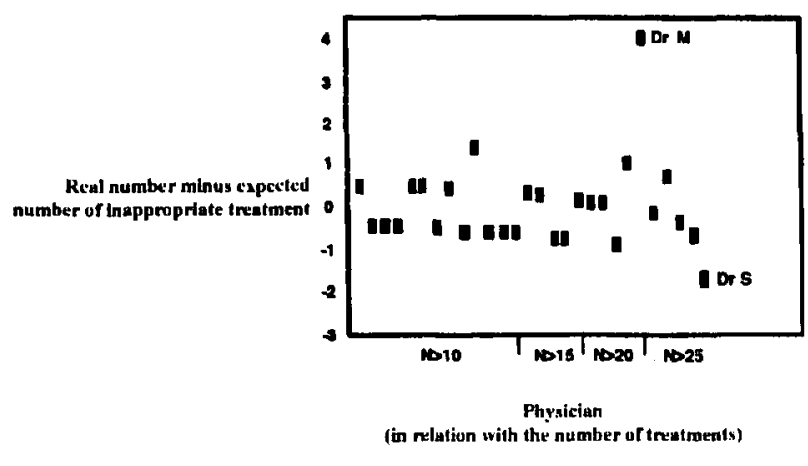

FIGURE 5 The statistical analysis of the medical performance allows the identification of certain tendencies. The statistics of Dr. M. indicate a mediocre performance that should be investigated and corrected if the investigation confirms the inappropriateness of his treatments. But the system will be much improved if the performance of the whole group is upgraded to that of Dr. S. whose outcomes are clearly superior to those of his colleagues. Adapted from Reference 6.

ing the criminal action will result in a restoration of the normally fluctuating balanced system. As they ensure the system stability, these activities are similar to QA. It has been suggested that for anaesthetic care, the system itself is the decisive factor rather than the attending anaesthetist himself. ${ }^{17}$ From this perspective, maintaining and improving the system are specially important.

The practice of QI will assist in identifying and correcting deficiencies. ${ }^{6}$ A subsequent assessment will be performed to check if the required changes have been implemented and if they are efficient. Figure 4 depicts the long term profit achieved by improving the system, compared with the individual correction of an immediate failure. Thus it is important to make sure that the changing results are not just caused by input changes (e.g., an older and weaker population as opposed to a younger and healthier population).

Figure 5 illustrates the usefulness of statistics and the necessity of interpreting them correctly and carefully. The diagram depicts the number of unsuitable treatments (vertical axis) in relation with the absolute number of treatments (horizontal axis) treated for a given pathology. As predicted, the mean for individuals approximates zero, i.e., that their level of performance was in agreement with expectancy. Two individuals clearly emerge, Dr. M. and Dr. S. According to this diagram, Dr. M.'s performance would have to be considered mediocre, but some special circumstances may have justified his behaviour. We must recognise that a statistical study can demonstrate the presence of a problem, but that it cannot identify what it is. In other words, the statistical study is an indicator and not a determinant of quality. As opposed to Dr. M., Dr. S. performance appears superior to that of his colleagues. If this can be confirmed by an investigation, the system will improve considerably if the global mean is upgraded to the level of Dr. S., instead of downgrading Dr. M. to the level of the group (this would also have to be done if the treatments were clearly inadequate).

\section{Management of resources}

The lack of quality is expensive in terms of loss of time for patients, physicians and hospital; in terms of loss of revenue and productivity for the employer; in terms of complications inside the hospital and outside and occasionally in terms of legal expenses. As a rule, high quality care is in everybody's interest.

One of the main objective of quality management is to conform the available resources to the requirements of health care. At present, budgetary compressions are straining our financial resources while technology has developed more effective drugs and highly performing equipment but it also generates higher costs.

In their study of four surgical operations involving moderate risk (renal transplant, caesarean section, total hip replacement, laparoscopic cholecystectomy), Dexter and Tinker have shown that, due to the present hign quality of anaesthesia, no additional advantages could be gained. ${ }^{18}$ They are only partially right. With the help of new drugs and more sophisticated monitoring, there is no doubt that anaesthesia has become remarkably safe. But from the viewpoint of the "customer," there is still ample room for improvement. For example, optimal anaesthesia will only briefly affect the patient operated for a minor procedure in short stay facilities and will involve lower cost for the individual, his family and his community. ${ }^{19}$

By itself, quality management is expensive and may be hard to justify on pure economical considerations. In another study performed by Eagle et al., cost efficiency was achieved in only four projects involved in quality management out of 53 and even then cost recovery didn't not provide for the autofinancing of the programme. ${ }^{20}$ Should such activities should be set aside? The answer is no, and for many reasons. ${ }^{21}$ Unlike the other specialities aiming at therapeutic goals, it is impossible in anaesthesia to measure cost savings achieved by QI using known indicators like quality adjusted life years (QALY). To measure cost efficiency achieved by QA, new tools specific to anaesthesia will have to be designed. Moreover, monetary benefits are less obvious when you work in environment where financial competition is poor.'

The consequences of non quality would be damaging to our speciality. Considering the increasing public 
interest in health care and the permanent implementation of budget restrictions, controls would be imposed from external bodies for the appraisal of practice using protocols of their own. In the US, numerous practice guidelines have been developed to reduce practice disparities between physicians and consequently to reduce health care costs. ${ }^{22}$ We are lucky to be able to practice in a system where the physicians are still autonomous. Only a practice of quality with economically accountable will preserve this freedom.

\section{Conclusions}

Quality management is a complex subject that must be approached from various perspectives. None of the above elements is essential to maintenance, continuity, improvement and cost management of quality.

This review invites each anaesthetist to look at quality management as a daily priority. In some way, it is the purpose ("why") of quality management of the professional practice. The implementation of the furthered principles ("how") will differ in conformity with the environment and/or the region of the anaesthetist. In Quebec, the reader is referred to the document published by the College of Physicians. ${ }^{3}$ This document correctly describes legal foundation, mechanisms and pertinent tools.

The quality in anaesthesia research is a different subject. Its impact is primary to clinical practice as much as fundamental and clinical research. Some mechanisms in use, like funding obrained from research agencies and peer review for submitted manuscripts, are variants of QA. ${ }^{23}$ However, in research, quality management needs more formal guidelines. ${ }^{23}$ The model described above could easily be adapted to research.

At the dawn of the third millennium, our objectives can be reached only through a constructive approach, patient oriented and appropriate to our environment i.e., to assure routinely and universally quality of anaesthesia in its domains, either technical, human or financial.

\section{References}

(See page R81) 